\title{
Atención odontológica de un paciente con autismo (tea), bajo el modelo psicoeducativo. Descripción de un caso*
}

Odontological care of a patient with autism (tea), under the psycheducational model. Case

Report

Atenção odontológica de um paciente com autismo (tea) através do modelo psiceducativo.

Relato de caso

Fecha de recepción: 21/06/2019 | Fecha de aceptación: 20/10/2019

\author{
Rocio ANDREA BARbosa ORJUEla \\ Universidad el Bosque. Bogotá, Colombia. \\ rbarbosao@unbosque.edu.co,hanabirocio@yahoo.com. https://orcid.org/0000-0003-1645-7964
}

*Descripción de caso.

aCorrespondencia: rbarbosao@ unbosque.edu.co,hanabirocio@yahoo.com

doi: https://doi.org/10.11144/Javeriana.uo38-81.aopa 
Cómo citar: Barbosa Orjuela RA. Atención odontológica de un paciente con autismo (tea), bajo el modelo psicoeducativo. Descripción de un caso. Univ Odontol. 2019 jun-dec; 38(81). doi: https://doi.org/10.11144/Javeriana.uo38-81.aopa

\section{RESUMEN}

Antecedentes: La Psicoeducación mejora las adaptaciones de las personas con autismo a nuevos ambientes o actividades a través de procesos de aprendizaje. Descripción del caso: Intervención interdisciplinaria para desarrollar habilidades y capacidades en un paciente con autismo - trastorno del Espectro Autista (TEA) - en la consulta odontológica. Paciente masculino de 19 años con diagnóstico Trastorno generalizado del desarrollo (TGD), autismo típico. Al examen clínico intraoral presenta placa bacteriana calcificada generalizada y lesiones iniciales de caries activas. Las intervenciones propuestas tienen como propósito mejorar comportamiento y ansiedad del paciente, potenciar la tolerancia en la consulta odontológica del paciente ante procedimientos bucodentales y mejorar las condiciones de salud bucal en el paciente. El tratamiento incluyo estrategias de anticipación, rutinas, extinción, ambientación del espacio en las que se programan, procedimientos de protección específica, periodoncia y operatoria básica. Conclusiones: El modelo propuesto en este documento para facilitar la atención odontológica de personas con TEA propone diversas estrategias dentro de las corrientes en psicología y educación especial. Las sesiones con apoyo de estrategias psicoeducativas culminan con resultados positivos para la salud bucal del paciente y un mejoramiento progresivo en el comportamiento del paciente.

\section{Palabras Clave}


autismo; discapacidad; modelamiento de comportamientos restrictivos; odontología; psicoeducación y TEA

\section{ABSTRACT}

Background: Psychoeducation improves the adaptations of people with autism to new environments or activities through learning processes. Case description: Interdisciplinary intervention to develop skills and abilities in the patient with autism TEA (Autism Spectrum Disorder) in the dental practice. 19-year-old male patient with diagnosis Generalized developmental disorder (TGD), typical autism. The intraoral clinical examination presents generalized calcified bacterial plaque and initial lesions of active caries. The proposed interventions are intended to improve patient behavior and anxiety, enhance tolerance in the dental practice of the patient before oral procedures and improve oral health conditions in the patient. The treatment included anticipation strategies, routines, extinction, setting of the space in which, specific protection, periodontics and basic operating procedures are programmed. Conclusions: The model proposed in this document to facilitate the dental care of people with ASD proposes various strategies within the currents in psychology and special education. Sessions with the support of psychoeducational strategies culminate with positive results for the patient's oral health and a progressive improvement in the patient's behavior.

\section{Keywords}

autism; disability; modeling restrictive behaviors; odontology; psychoeducation and TEA 


\section{RESUMO}

Antecedentes: A psicoeducação melhora as adaptações de pessoas com autismo a novos ambientes ou atividades por meio de processos de aprendizagem. Descrição do caso: intervenção interdisciplinar, durante a consulta odontológica, com o objetivo de desenvolver habilidades e capacidades em um paciente com autismo - Transtorno do Espectro do Autismo (TEA) -. Paciente do sexo masculino, 19 anos, diagnosticado com Transtorno Global do Desenvolvimento (TGD), autismo típico. Ao exame clínico intraoral apresenta placa bacteriana calcificada generalizada e lesões iniciais de cárie ativa. O objetivo das intervenções propostas foi melhorar o comportamento e a ansiedade do paciente, aumentar a tolerância do paciente à prática odontológica durante procedimentos orais e melhorar as condições de saúde bucal do paciente. O tratamento incluiu estratégias de antecipação, rotinas, extinção, adaptação do consultório onde estão agendados os procedimentos de proteção específica, periodontia e dentística operatória. Conclusões: o modelo proposto neste documento, para facilitar o atendimento odontológico de pessoas com TEA, é composto de várias estratégias dentro das correntes da psicologia e da educação especial. As sessões com o apoio de estratégias psicoeducacionais culminaram em resultados positivos para a saúde bucal do paciente e uma melhoria progressiva no comportamento do paciente.

\section{Palavras-Chave}

deficiência; modelagem de comportamentos restritivos; odontologia; psicoeducação; transtorno autístico; transtorno do Espectro do Autismo

\section{INTRODUCCIÓN}


Los trastornos del espectro autista (TEA) son un grupo de alteraciones del neurodesarrollo de origen biológico y neurológico que provocan restricciones en la comunicación social, comportamientos e intereses individuales y sociales (1). El TEA es una condición que dura toda la vida del individuo, su prevalencia es de 1 por cada 68 personas y afecta cuatro veces más al género masculino que el género femenino, un tercio de los casos presentan asociación con discapacidad cognitiva o intelectual (2). Las personas con TEA requieren terapias psicosociales durante toda su vida como apoyo para su participación familiar y social. El TEA no tiene una etiología clara, se ha asociado a factores genéticos, epigenéticos (3) y ambientales. El Manual diagnóstico y estadístico de los trastornos mentales (DSMV) (1) describe el TEA como una dificultad en la comunicación e integración de la comunicación verbal y no verbal, aunque cada caso tiene sus particularidades y no se puede generalizar al diseñar estrategias o apoyos. El diagnóstico se realiza de acuerdo con teorías conductuales y comportamentales, ya que por su etiología heterogénea en la actualidad se carece de pruebas de laboratorio precisas para un diagnóstico concluyente (4).

Las dificultades más comunes en personas con TEA son la comunicación, la expresión verbal, las alteraciones sensoriales, inexpresividad y la escasa adaptación a los cambios. Frente a intereses restringidos o conductas repetitivas algunas personas con TEA presentan conductas de inflexibilidad, irritabilidad y rituales o estereotipias motoras o verbales (5).

Los rituales, estereotipias o autoestimulaciones en las personas con TEA se refieren a una repetición o simetría de una acción o sonido verbal (llamado ecolalia) o no verbal, generalmente involucra un orden y número de veces, Dentro de las estereotipias se incluyen movimientos 
motores extravagantes, como aleteo de manos o brazos, agitación de objetos, balanceo rítmico y rotación de la cabeza, andar de puntillas, dar saltos, adquisición de posturas peculiares, hacer carreras, medición de pasos y muchas otras (6).

Estas estereotipias bajan los umbrales de ansiedad o estrés ante situaciones no anticipadas o acciones que generan molestia o irritabilidad. Algunas de estas estereotipias pasan desapercibidas en los individuos con mayor funcionalidad social y comunicacional. Las personas con TEA también pueden presentar alteraciones sensoriales en las cuales podría existir hipersensibilidad visual, sensitiva, auditiva, táctil u olfativa, estas pueden provocar malestar o escasa tolerancia. En otras ocasiones se presenta hiposensibilidad al calor, al dolor y al apetito o hipofagia, lo que puede ocasionar peligro para su condición de salud o vida.

En las personas con autismo se observan habitualmente aspectos de un juego rígido limitado y ritualizado, en general organizan obsesivamente las piezas por textura, forma, color o forjan obsesiones por colección de un tipo específico de objetos o juguetes. No generan variedad en sus juegos, además muchas veces crean un apego inusual por algunos objetos con las cuales componen estereotipias. Todas estas condiciones o características de las personas con TEA pueden presentarse conjugadas de diferentes formas, lo que hace complejo el trastorno e indeterminado (7). Por otra parte, se reportan para las personas con TEA en algunos casos comorbilidades con Trastorno de déficit de atención y/o hiperactividad, (TDAH) esta condición afecta el funcionamiento de la memoria de trabajo ejecutivo, además se incrementa en ellos la irritabilidad y excitabilidad (7). 
En general el trastorno del espectro autista (TEA) presenta una etiología y unas características heterogéneas, lo que implica el diseño de diferentes herramientas y apoyos para el abordaje de cada caso, así como identificar sus características identitarias y particulares como persona, en consecuencia con su etapa de curso de vida y ámbitos sociales.

La Psicoeducación (8) se propuso como metodología de intervención a mediados del siglo XX para las enfermedades mentales; esta estrategia reúne acciones interdisciplinarias desde la psiquiatría, psicología, pedagogía, educación y trabajo social. El programa psicoeducativo incluye el trabajo con la persona y su familia o personal de apoyo, frente al significado de la enfermedad o condición a intervenir, identificar sus causas, síntomas iniciales, desencadenantes y de forma participativa diseñar estrategias que minimicen comportamientos inadecuados.

Desde las metodologías de Psicoeducación se mejoran las adaptaciones de las personas con autismo a nuevos ambientes o actividades a través de procesos de aprendizaje. En estos procesos se estimulan habilidades de autocontrol y conductas adaptativas. Una condición importante de estas metodologías psicoeducativas es el aporte como modelo educativo muy alternativo al conductismo clásico, en el cual sólo se limitaba a eliminar conductas inadecuadas, pero sin generar un cambio alternativo desde el aprendizaje, entendimiento y desarrollo de potencialidades del individuo (8). Los principios técnicos que se deben tener en cuenta para el proceso de aprendizaje en adaptabilidad de una persona con autismo son: 1. Eliminar los estímulos desencadenantes de las conductas disruptivas. 2. Enseñar estrategias y habilidades básicas para controlar las situaciones desencadenantes de las conductas. 3. Fomentar el uso de conductas adaptadas y adecuadas a esas situaciones. 4. Suprimir cualquier tipo de refuerzo antecedente o posterior, 
positivo o negativo, que sea contingente a la conducta disruptiva y la pueda mantener o incrementar (9). Además, las estrategias implementadas en el proceso de adaptabilidad deben seguir unas instrucciones específicas, entre ellas: 1. Ofrecer actividades rutinarias y asequibles al nivel de desarrollo y que posean, a su vez, un alto grado de estructuración y que posibiliten el proceso de aprendizaje que se quiera fomentar entre estos adaptabilidades o anticipaciones. 2. Generar oportunidades para practicar, ensayar y poder generalizar las conductas adaptativas a otros contextos y situaciones. 3. Enseñar técnicas de autocontrol para reducir la ansiedad. 4. Entrenar las respuestas a posibles cambios del entorno y de sus interacciones. 5. Modificar las conductas desafiantes y agresivas por conductas positivas, reemplazando los comportamientos disruptivos por otros más aceptables y adaptativos. 6. Usar, de modo generalizado, los distintos contextos educativos para fortalecer los cambios de conducta. Y 7. Atender y valorar los síntomas conductuales asociados que pueden requerir un plan de intervención individualizado (9).

La atención odontológica para pacientes con autismo requiere componentes interdisciplinarios, familiares, de apoyo técnico y psicoeducativo. Luego de la revisión temática se identifican algunas metodologías de orden terapéutico y educativo para mejorar los comportamientos y la comunicación de este tipo de pacientes, características que requieren modelamiento para lograr una atención odontológica optima que logre mantener la salud bucal de los pacientes con autismo. Sin embargo desde los modelos educativos o psicoeducativos es escaso el abordaje en las ciencias odontológicas.

El objetivo del presente artículo es divulgar el modelo psicoeducativo como estrategia para el modelamiento de conductas en odontología. En la actualidad se proponen solo modelos 
psicológicos o alternativas de sedación o anestesia general para manejar conductualmente pacientes con TEA. La alternativa propuesta en este artículo permite modelar conductas restrictivas que impiden el desarrollo natural de una consulta en odontología.

\section{DESCRIPCIÓN DEL CASO}

El modelo propuesto en este reporte incluye intervención interdisciplinaria de profesionales odontólogos, psicólogos y educadores especiales, para desarrollar habilidades y capacidades en el paciente con TEA en la consulta odontológica. Las herramientas y acciones se programan para el paciente desde el modelo psicoeducativo además se refuerzan en los espacios hogar y educativo continuamente, con una semana anticipada a la consulta, estas acciones se soportan en las estrategias descritas a continuación:

1. ABA análisis del comportamiento aplicado (10) por su sigla en inglés Applied Behavior Analysis es una metodología que tiene como objetivo aplicar los principios de aprendizaje de forma continua y sistemática para incrementar, disminuir o mantener conductas o comportamientos de personas con condiciones cognitivas, intelectuales o con autismo.

2. Teacch (11) por su sigla en inglés Treatment and Education of Autistic and related Communication- handicapped Children es una herramienta que posibilita a través de sus estrategias la comunicación e interacción con personas que presentan restricciones sociales y de la comunicación. Teacch aplicado continuamente permite a la persona entender el mundo, el contexto de vida y vivir lo más independientemente posible. 
3. Son-Rise(12) es un programa desarrollado para tratar personas con TEA con implementación en el hogar y espacio educativo, debe ser desarrollado en un ambiente natural, libre de distracciones y enfatiza en potenciar las interacciones sociales.

Inicialmente se toman los repertorios de análisis conductual de ABA (10), para esto es necesario tener el tiempo, mínimo en una o dos consultas en las cuales se pueda realizar una observación directa del paciente con relación a la interacción y comunicación con el medio donde se desarrolla la consulta. Se propone en esta primera sesión, diligenciar documentos como historia clínica del paciente y otros instrumentos que permitan evaluar la comunicación e interacción del paciente y las conductas problema que puedan presentarse, además de familiarizar el paciente con los elementos propios de un consultorio odontológico. Para esto se debe apoyar el proceso en la estrategia "Decir, mostrar, hacer" (13) en la cual se muestra y acciona cada equipo del consultorio, para que el paciente conozca las dinámicas naturales de una consulta odontológica. Esta estrategia se llama en el análisis de comportamiento aplicado ABA; anticipación, en la cual se prepara al paciente frente a las situaciones que causan estrés, con tal de que él modele conscientemente sus reacciones naturales de miedo y desconocimiento. Las categorías de análisis según los repertorios ABA que deben analizarse en el paciente y que son de importancia para el desarrollo de una consulta odontológica son: 1. Conductas Básicas: atención, imitación motora, seguimiento de instrucciones. 2. Habilidades sociales y de adaptación: lenguaje de comunicación, conducta motora gruesa y conducta motora fina. 3. Conductas problemáticas: auto estimulación física, auto estimulación vocal, hiperactividad, interrumpir a otros, falta de control a impulsos y autolesiones. El análisis e intervención de todas estas conductas son necesarias para el desarrollo de una consulta odontológica y el cuidado bucal. Las conductas que no presenten una evaluación 
positiva deben tener apoyos para potenciar estas habilidades. Los apoyos que se diseñan para los casos se soportan en las herramientas Teacch y Sun-rise. La estrategia o método Teacch (11) fue desarrollado por el profesor Eric Schopler en la Universidad de Carolina del Norte a principio de los años 70. TEACCH es un método patentado por la Universidad de Carolina del Norte en Chapel Hill y realiza sus intervenciones en clínicas de servicios regionales en Carolina del Norte, además tiene en todo el mundo terapeutas oficiales que aplican sus estrategias. El programa Sun Rise (12) se implementó y reporto en el año 1979 por el matrimonio Kaufman, ellos como padres de un hijo con autismo decidieron observar sus conductas y probar diferentes estrategias para mejorar los comportamientos disruptivos de su hijo. Luego de varios años de análisis y aplicación de estrategias continuas, lograron que su hijo mejorara la interacción y participación socio familiar, a través de intervenciones familiares con intensidades progresivas y continuas.

Las herramientas Teacch (11) buscan potenciar las habilidades y destrezas de las personas con TEA con tal de mejorar sus dinámicas de vida diaria. Para esto se fortalecen sus habilidades de comunicación, socialización y procesos de autonomía, reconociendo sus capacidades individuales y diferenciales. Las acciones Teacch potencian además las habilidades de contacto visual, concentración, comprensión, atención, desplazamiento y adaptación. Por otra parte, permite controlar los impulsos exagerados de respuesta y la intolerancia a ciertas situaciones que puedan presentarse sin anticipación. Para desarrollar esta estrategia se diseñan apoyos visuales donde se anticipen las acciones que deben realizarse en tareas discriminadas y con uso de pictogramas, esto con el fin de reforzar en su proceso de pensamiento la acción que debe ejecutarse y programar los acontecimientos que pueden suceder durante la consulta. Siempre debe estar en uso el factor "Predictibilidad" para anticiparse a cualquier situación y preparar al paciente frente a esta, con el 
objetivo de generar confianza y escasos sucesos desconocidos para él, que puedan ocasionar ansiedad o intolerancia. La comunicación continua con el paciente durante el desarrollo de la consulta es otro factor indispensable, siempre se debe utilizar la herramienta "Decir, mostrar, hacer" para reforzar la anticipación en el curso de la consulta. Para el desarrollo de estas estrategias se requiere un apoyo familiar y profesional continuo, trabajando la anticipación en el hogar y el ambiente educativo o terapéutico con el uso de agendas visuales, las cuales son apoyos visuales a través de imágenes o pictogramas para orientar orden, forma y secuencia de una actividad específica, estas agendas deben contener cada una de las acciones o tareas de forma discriminada, paso a paso; además se reforzar la socialización de la consulta odontológica. En algunas ocasiones es necesario el uso de apoyos de desensibilización frente a algunas acciones que afectan el proceso sensorial del paciente, el caso específico de luces, colores, ruidos o sensaciones táctiles que generen hipersensibilidad en el paciente. Para esto se requiere de igual forma el apoyo familiar y terapéutico continuo con aproximaciones sucesivas que generen la adaptabilidad de esa situación en el paciente.

Cada una de estas acciones buscan generar en la persona con autismo la tolerancia, el autocontrol sobre la conducta y potencia su autonomía frente a sus capacidades. De acuerdo con las estrategias Teacch es necesario tener en cuenta la ambientación del espacio de trabajo para la consulta odontológica, para esto debe tenerse en cuenta la potencialidad sensorial del paciente y evitar aquellos objetos o acondicionamientos que generen temor, intolerancia o novedad en el paciente. La comunicación al inicio de la cita odontológica debe contar con un intérprete que puede ser el profesional acompañante o el cuidador principal, que mejore la comunicación entre el paciente y el odontólogo, en el proceso y con desarrollo de comunicación aumentativa se pretende mejorar la 
interacción comunicativa entre el odontólogo y el paciente con TEA. También se deben agendar los horarios y sistemas de trabajo con las anticipaciones necesarias en el paciente por lo menos una semana previa. Todas estas acciones van encaminadas a generar una conducta adaptativa del paciente a la consulta odontológica.

Las técnicas de abordaje Son-Rise (12) para paciente con TEA contemplan mejorar la relación e interacción de la persona con autismo y su entorno (otras personas y los ambientes o espacios); esto se potencia a través de procesos de aprendizaje continuo, conexión emocional, flexibilidad y motivación de aprendizaje. Las herramientas útiles de este programa para la consulta odontológica se soportan en tomar las conductas problema como estereotipias o movimientos repetitivos que presenta el paciente y utilizarlas para lograr una conexión con el paciente. Para esto se debe contar con tiempo ya que durante varias sesiones el profesional debe imitar los movimientos repetitivos o estimulatorios del paciente, estos movimientos lo que causan en la persona con autismo es lograr un autocontrol sobre lo que está causando ansiedad, al imitar el movimiento o estereotipia se logra generar una relación a través de un interés común, de esta forma se crea un vínculo de aceptación y protección que mejora la comunicación y confianza, esto puede en gran medida facilitar cualquier actividad conjunta con la persona con autismo. Esta herramienta puede potenciar la participación del paciente en la consulta y permitirle el principio de autonomía sobre la misma. Para esto el profesional debe ser muy flexible frente a las acciones que se deben realizar y planear muy bien cada uno de los procedimientos. Las acciones que se desarrollan para el uso de esta herramienta son: inicialmente No mantener una comunicación oral, solo permitir la comunicación visual directa con el paciente, el paciente puede sentir incomodidad y ser intolerante ante esta acción, pero debe intentarse con continuidad mantener el contacto visual, hasta lograr un espacio 
prolongado de este. En segunda medida y luego de lograr esta primera interacción, se debe iniciar la comunicación verbal a través de la expresión oral de objetos o instrucciones, de esta forma la persona con TEA logra interpretar los mensajes, esto debe tener una continuidad con tal de avanzar progresivamente y de acuerdo con las individualidades del paciente, esto se desarrolla a través de las neuronas en espejo, condición reportada en este tipo de personas (12). En tercera medida se inicia el proceso de imitación, aquí se potencia la concentración de la persona con TEA al lograr un interés común. Cuando se logren largos periodos de interacción con el paciente se intentan cambios de actividad bruscos con tal de generar cierta tolerancia y flexibilidad. Cada una de estas acciones muestra pequeños progresos en prolongados tiempos, por eso esta herramienta solo puede usarse en casos en los cuales se compartan espacios comunes con el paciente como instituciones donde brinden apoyos de desarrollo continuo para su proceso, pero a pesar del gran esfuerzo por parte del terapeuta o profesional se logran avances que mejoran la relación con los ambientes y pueden tolerar todo tipo de procedimientos, para el caso de atención odontológica, con el apoyo de las otras técnicas mencionadas en este documento.

Luego del análisis conductual individual se requieren ajustar las acciones que deben aplicarse con el paciente de forma individual de acuerdo con los tiempos, procedimientos odontológicos necesarios y apoyo terapéutico y familiar. Cada caso requiere una programación personalizada y debe evaluarse continuamente por el equipo interdisciplinario (14).

\section{Diagnóstico}

El objeto de este reporte es plasmar el caso de un paciente masculino de 19 años con diagnóstico confirmado Trastorno generalizado del desarrollo TGD, autismo típico según clasificación CIE10, 
trastorno autista según DSMV, a nivel sistémico no presenta diagnósticos asociados. El paciente vive con sus padres y recibe terapias de psicología, lenguaje, ocupacional, fisioterapia y educación espacial una vez a la semana. Al realizar la caracterización de comportamiento del paciente a través de repertorios ABA (10) se identifica tendencia al aislamiento, ecolalia (Te, te, te...), conductas repetitivas con las manos, intolerancia moderada a la luz y los sonidos fuertes, movimientos de balanceo e hiperactividad, ante instrucciones específicas no hay seguimiento y presenta escaso lenguaje oral y corporal. Paralelamente se identifica un interés estereotipado por tocar todos los objetos, fascinación por objetos redondos o de movimiento giratorio.

Al realizar el examen bucodental se utilizan herramientas ABA (10) y Teacch (11) con el fin de lograr tolerancia en el paciente. Durante las sesiones se utiliza la herramienta de anticipación para cada una de las acciones, decir, mostrar y hacer antes de cualquier actividad, para permitir al paciente familiarizarse con el consultorio y cada uno de sus equipamientos, sonidos, imágenes y luces. Se familiariza al paciente con un reloj, en el cual se le explica la duración de la consulta de acuerdo con el movimiento de las manecillas; de acuerdo con los repertorios anticipatorios Teacch (11), también se hace uso de agendas visuales con pictogramas sobre la asistencia en la consulta. Esta agenda debe tener reforzamiento en el hogar. Luego de la tercera cita se logra realizar el examen odontológico en el cual se evidencia la presencia de placa dura generalizada, caries de mancha blanca en 24, 25 y 45 por oclusal clasificación ICCMS (15) CP1, pérdida superficial de esmalte con sombra subyacente en 36 CP4. Además, se identifica un mejoramiento progresivo del comportamiento en el paciente, es más tolerante a cada una de las acciones, no expresa ansiedad y permite realizar actividades en su cavidad oral. 


\section{Objetivos}

Mejorar comportamiento y ansiedad del paciente con aproximaciones sucesivas y anticipación. Indicaciones a padres y terapeuta para realizar técnicas operantes como refuerzos positivos o negativos ante acciones de autocuidado bucal con apoyos. - Tolerancia en la consulta odontológica del paciente ante procedimientos bucodentales. - Mejorar las condiciones de salud bucal en el paciente.

\section{Plan de acción}

Luego de realizar el diagnóstico integral durante 2 meses en citas semanales de 45 minutos se decide diseñar un plan de tratamiento integrativo, en el cual se utilicen estrategias de anticipación, rutinas, extinción, la cual hace parte de las estrategias de condicionamiento clásico de la psicología, en la cual se hace omisión de atención frente a una conducta negativa en la persona, además es necesario realizar ambientación del espacio minimizando la decoración, colores neutros y espacios adecuados para movimientos amplios, se debe apoyar las acciones con objetos redondos como pelotas suaves, para el uso de las manos del paciente durante la consulta y agendas visuales con tareas discriminadas con reforzamiento diario en el hogar para mejorar tolerancia durante los procedimientos. Las agendas visuales se diseñaron individualizadas con fotos e imágenes simples o pictogramas de cada una de las tareas a realizar durante la consulta, estas agendas fueron impresas y plastificadas en algunas ocasiones, aunque en otras se usó la agenda digitalizada. Referente a las necesidades bucodentales se programan procedimientos de protección específica, periodoncia y operatoria básica. Además, se diseña un plan casero para el autocuidado bucal con apoyos. 
Para los procesos de aprendizaje con personas con autismo es útil identificar sus intereses con el fin de motivar y generar una respuesta positiva y selectividad autónoma del individuo en el proceso. Si no se logra captar el interés del individuo puede aparecer una limitación en la adquisición de nuevos aprendizajes que favorezcan la adaptación. Las estereotipias pueden ser usadas como reforzadores en los procesos de aprendizaje o adaptación a ambientes o actividades. Por tanto, se diseña el plan de tratamiento en un curso de adaptación con el paciente como proceso de aprendizaje con componentes de continuidad, conexión emocional, motivación y reforzamientos positivos o negativos a través de la voz. Se utilizan estrategia Son- Rise de imitación ante ecolalias para mejorar comunicación, confianza y flexibilidad.

La atención odontológica cursó en 6 sesiones de 35 minutos, en las primeras 3 sesiones se realizaron acciones de diagnóstico y adaptación del paciente. Los procedimientos bucodentales se realizaron durante 3 sesiones más: durante la primera sesión se realizó procedimiento de profilaxis dental con micromotor y detartraje supragingival con Cavitrón en primer cuadrante. En la segunda sesión se terminó detartraje supragingival en los cuadrantes 2,3 y 4. Y se procedió a aplicar topicación de flúor en barniz. En la última sesión se procedió a realizar obturación con ionómero de vidrio en el diente 36 por oclusal y educación para el autocuidado bucodental del paciente con apoyo de sus padres.

Las sesiones en las que se utilizaron instrumentos rotatorios como piezas de mano, Cavitrón o micromotores con sonido y movimiento, el paciente inicialmente presentó desadaptación e intentaba pararse del sillón, pero al dar indicación procedía a continuar sentado y tolerando el procedimiento, por aproximadamente 5 minutos y continúa de esta forma cada sesión hasta 
culminar el plan de tratamiento. Las sesiones culminan en este período con resultados positivos para la salud bucal del paciente y un mejoramiento progresivo en el comportamiento del paciente. El paciente asistió a consulta de diagnóstico integrativo e intervención en odontología por un periodo de tres meses y medio, posteriormente se programa para seguimiento y control a los tres meses. Esto con el objetivo de realizar reevaluación, fase higiénica bucodental y aplicación de flúor en barniz. Se solicita a los padres realizar en casa "anticipación” por lo menos una semana antes de la consulta con apoyo de agendas visuales personalizadas y tareas discriminadas para facilitar el proceso. Agenda diseñada y construida de forma personalizada con fotos reales del consultorio, objetos y personal operativo, entre estos el profesional en odontología.

\section{DISCUSIÓN}

El modelo propuesto en este documento para facilitar la atención odontológica de personas con Autismo TEA, propone diversas estrategias dentro de las corrientes en psicología y educación especial. En este mismo sentido las estrategias psicoeducativas (16) proponen mejorar comportamientos disruptivos y aceptabilidad en el desarrollo de las consultas odontológicas para este tipo de pacientes. Varios estudios de caso (17-19) han reportado dificultades específicas en áreas sociales, comunicativas o de lenguaje y movimientos repetitivos o estereotipias que dificultan la atención en odontología de pacientes con autismo TEA. Para esto proponen diversas estrategias (17-19) de modelamiento conductual pero no se puntualiza en un modelo específico para aplicación conductual durante la consulta. 
Otros autores como Rouches y Col (20) proponen los modelos conductuales ABA y Teacch para facilitar el comportamiento de los pacientes con Autismo durante la consulta odontológica. Al igual que en este reporte de caso clínico, los autores reportan conductas positivas y tolerancia a los procedimientos con progresividad de acuerdo a la continuidad de las estrategias. El modelo propuesto en este artículo además de las técnicas propuestas por Rouches y Col (20) propone el modelamiento como una técnica de aprendizaje bajo el modelo psicológico y académico con apoyo de la Psicopedagogía.

Sin embargo, tal y como es reportado por Virues- Ortega y Col (21) las estrategias Teacch de acuerdo su investigación, son modelos conductuales que requieren continuidad y repetición para mejorar los comportamientos sociales y regular la adaptación y tolerancia de las personas con Autismo durante actividades generadoras de estrés o ansiedad.

\section{CONCLUSIONES}

Para el caso reportado las técnicas decir-mostrar- hacer, manejo de voz, restricción física y refuerzos positivos y negativos no son suficientes para el desarrollo de una atención odontológica con un paciente con autismo, es necesario incluir estrategias como análisis conductual aplicado, anticipaciones, extinciones, análisis del lenguaje, diseño de lenguaje, desarrollo de autonomías, capacidades, habilidades y ambientación de espacios, entre otras, técnicas desarrolladas en las metodologías ABA, Teach y Sun-rice utilizadas en áreas de educación, psicología y comunicación. En el presente artículo se divulga el modelo psicoeducativo para el modelamiento de conductas de pacientes con TEA en la atención odontológica a partir del caso reportado, se sugiere con 
posterioridad dar continuidad al reporte de casos con este tipo de intervenciones y realizar investigaciones con evaluaciones de índole psicológicas que soporten el cambio conductual y comportamental de los pacientes.

\section{AGRADECIMIENTOS}

Agradecimientos a la Universidad el Bosque por apoyar el trabajo e investigación en las ciencias odontológicas para personas con condiciones diferenciales, a la facultad de odontología y sus directivas por brindar apoyo y motivación en la tarea del trabajo para poblaciones con necesidades especiales.

\section{REFERENCIAS}

1. American Psychiatric Association (APA). Diagnostic and statistical manual of mental disorders (DSM-5). Fifth edition. Washington DC, United States: APA; 2013.

2. Van Naarden Braun K, Christensen D, Doernberg N, Schieve L, Rice C, Wiggins L, Schendel D, Yeargin-Allsopp M. Tendencias en la prevalencia del trastorno del espectro autista, parálisis cerebral, pérdida de audición, discapacidad intelectual y discapacidad visual. Atlanta metropolitana, 1991-2010. Más uno. 2015; 10 (4).

3. Lyall K, Schmidt RJ, Hertz-Picciotto I. Maternal lifestyle and environmental risk factors for autism spectrum disorders. Int J Epidemiol 2014; 43(2): 443-64

4. Lorenz K. King Solomon's ring: new light on animal ways. Routledge, London. 2002. 
5. Hervás A. "Un autismo, varios autismos. Variabilidad fenotípica en los trastornos del espectro autista [XVIII Curso Internacional de Actualización en Neuropediatría y Neuropsicología Infantil]." Revista de Neurología. 2016; 2(1): 9-S14

6. Durand VM, Carr EG. Social influences on "self-stimulatory" behavior: analysis and treatment application. J Appl Behav Anal. 1987 Summer; 20(2): 119-32

7. Nazeer A, Ghaziuddin M. Autism spectrum disorders: clinical features and diagnosis. Pediatr Clin North Am. 2012; 59(1): 19-25

8. Association International Autisme Europe (AIAE) (2000). Description de 1 Áutisme, document prepare sous les auspicies do "Conseil dÁdministration dÁutisme Europe". Bruselas: AIAE.

9. López Gómez, Santiago, and Consuelo García Álvarez. "Patrones comportamentales en el trastorno autista: descripción e intervención psicoeducativa." Educational Psychology. 2007; 13(2): 117-31.

10. Bostow, D and Bailey, J. Modification of severe disruptive and aggressive behavior using brief timeout and reinforcement procedures. Journal of Applied Behavior Analysis ABA. 1969; 2(1): $31-37$.

11. Callahan K, Shukla-Mehta S, Magee S, Wie M. ABA versus TEACCH: the case for defining and validating comprehensive treatment models in autism. J Autism Dev Disord. 2010; 40(1): $74-88$

12. Zink AG. Novo método de paciente autista. Rev APCD. 2011; 32: 10-11.

13. Gutiérrez, Laura; Ramírez, Jennifer. Estrategias pedagógicas aplicadas en el proceso de aprendizaje de personas con discapacidad cognitiva. Revista umbral científico. 2012; 21(9): p20-28. 
14. Sanz López, Yolanda, Teresa Guijarro Granados, and Vicente Sánchez Vázquez. "Inventario de Desarrollo Battelle como instrumento de ayuda diagnóstica en el autismo." Revista de la asociación española de neuropsiquiatría. 2007; 27(2): 31-45.

15. Pitts, NB, KR Ekstrand y la Fundación ICDAS. "El Sistema Internacional de Detección y Evaluación de Caries (ICDAS) y su Sistema Internacional de Clasificación y Manejo de Caries (ICCMS, por sus siglas en inglés): métodos para organizar el proceso de caries y permitir que los dentistas manejen la caries". Odontología comunitaria y epidemiología oral. 2013; 41(1): e41-e52.

16. Orellana, Mirtala. Intervencion Psicoeducativa para facilitar el examen clínico odontológico de personas con TEA. Tesis Doctoral. Universidad de Valencia. Facultad de medicina. Departamento de estomatología. 2013.

17. Alaniz Paredes Astrid, Alvear-Córdova María Cristina, Paredes Andrés. Tratamiento estomatológico multidisciplinario de un paciente con trastorno de espectro autista: reporte de un caso. Revista OACTIVA UC Cuenca. Enero-Abril, 2017; 2(1): 43-50.

18. Oliva, Jacqueline. La comunicación en la atención estomatológica en niños autistas. Comunicación Social: retos y perspectivas. Centro de Lingüística Aplicada, Santiago de Cuba. 2015

19. Betancourt, Carlos Raúl. Atención diagnóstica y tratamiento odontológico primario del paciente autista en la consulta odontopediátrica. Trabajo de grado. Universidad de Guayaquil. Facultad Piloto de odontología. 2013

20. Rouches, A., et al. "Amélioration de la santé orale des enfants avec autisme: les outils à notre disposition." Archives de Pédiatrie. 2018; 25(2): 145-49. 
21. Virues-Ortega, Javier, Flávia M. Julio y Roberto Pastor-Barriuso. "El programa TEACCH para niños y adultos con autismo: un metaanálisis de los estudios de intervención". Revisión de psicología clínica. 2013; 33(8): 940-53. 